\title{
Preferential radiosensitization to glioblastoma cancer stem cell-like cells by a Hsp90 inhibitor, N-vinylpyrrolidone-AUY922
}

\author{
TOSHIAKI TANI ${ }^{1}$, NAOMI TOJO ${ }^{2}$ and KEN OHNISHI ${ }^{2}$ \\ ${ }^{1}$ Radiological Technology Section, QST Hospital, National Institutes for Quantum and Radiological Science and Technology, \\ Chiba 263-8555; ${ }^{2}$ Department of Biology, Ibaraki Prefectural University of Health Sciences, Inashiki, Ibaraki 300-0394, Japan
}

Received August 12, 2021; Accepted December 22, 2021

DOI: $10.3892 / \mathrm{ol} .2022 .13222$

\begin{abstract}
The present study examined the radiosensitization induced by a heat shock protein 90 inhibitor, $\mathrm{N}$-vinylpyrrolidone (NVP)-AUY922, in CD133-positive cells in a hypoxic area of T98G spheroids. CD133-positive cells that are induced in the hypoxic microenvironment of spheroids have previously been reported to exhibit cancer stem cell-like properties. The present study used CD133-positive cells from a glioblastoma cell line (T98G) as cancer stem cell-like cells. CD133-positive and negative cells were sorted from T98G spheroids using fluorescence-activated cell sorting and used for colony formation assay. Colony formation assay results indicated that NVP-AUY922 enhanced radiosensitivity more strongly in CD133-positive cells compared with CD133-negative cells This result showed that NVP-AUY922 was a preferential radiosensitization candidate targeting glioblastoma cancer stem cells. The mechanisms underlying radiosensitization by NVP-AUY922 are discussed in relation to the properties of cancer stem cells. Overall, HIF-1 $\alpha$ inhibition by NVP-AUY922 may induce higher sensitization of cancer stem cells to radiation.
\end{abstract}

\section{Introduction}

Glioblastoma multiforme (GBM) is particularly refractory among tumors and is highly resistant to radiation, with a 5 -year survival rate of $10 \%$ or less (1). The average life expectancy of GBM patients is 1-2 years. GBM diffuses and invades the normal brain tissue; therefore, it is difficult to surgically remove the tumor completely. Some cell fractions have been reported to be highly resistant to cancer therapy. Some of these cells are categorized as cancer stem cells (CSCs). Even if the tumor shrunk or seemingly disappeared after treatment with radiation or powerful anti-cancer drugs, surviving CSCs are

Correspondence to: Professor Ken Ohnishi,Department of Biology, Ibaraki Prefectural University of Health Sciences, 4669-2 Ami, Inashiki, Ibaraki 300-0394, Japan

E-mail: ohnishi@ipu.ac.jp

Key words: cancer stem cell, N-vinylpyrrolidone-AUY922, heat shock protein 90 inhibitor, glioblastoma, radiosensitization thought to cause recurrence or metastasis. Additionally, CSCs have been reported to cause difficulty in GBM therapy (2-6).

Our previous report (7) showed that cells immunofluorescently positive for CD133, a marker of glioblastoma stem cells, are induced in the hypoxic microenvironment of spheroids, which are three-dimensional culture models (8). Furthermore, we revealed that spheroid CD133-positive cells (SCPCs) exhibit some CSC-like characteristics, including increased migration and inversion abilities and resistance to radiation and anti-cancer agents (9). Based on these results, we propose that SCPCs are glioblastoma CSC-like cells, as shown in Fig. 1. Using SCPCs, the present study examined whether the sensitivity of CSC-like cells to radiation was enhanced by N-vinylpyrrolidone (NVP)-AUY922.

Heat shock protein 90 (Hsp90) is a well-known chaperone protein that protects against the degradation of numerous client proteins that contribute to the maintenance of cancer cells (10). Inhibition of Hsp90 function may lead to the loss of cancer cell malignancy. Thus, targeting Hsp90 using Hsp90 inhibitors provides a promising strategy to develop anti-cancer agents $(11)$ or radiosensitizers $(12,13)$. Geldanamycin and its derivative 17-allylamino-17-demethoxygeldanamcyin (17-AAG) are commonly used Hsp90 inhibitors (14). However, it has been reported that Hsp90 inhibitors cause severe side effects concerning the kidney and liver and chemoresistance in some tumors, including GBM (15-17). NVP-AUY922 is a purine-scaffold derivative and non-geldanamycin analog of 17-AAG (18). NVP-AUY922 mimics the ATP/adenosine diphosphate (ADP)-binding interface of the Hsp90 N-terminus, thereby inhibiting client proteins. NVP-AUY922 effectively overcomes 17-AAG resistance in glioblastoma cells with less pronounced side effects, even at low doses (19). In the present study, we examined the radiosensitizing effects of NVP-AUY922 on SCPCs and spheroid CD133-negative cells (SCNCs) derived from spheroids cultured from T98G cells. Radiosensitization by Hsp90 inhibitors has not yet been examined in preclinical studies targeting cancer stem cells. The novelty of the present study is to compare effects of NVP-AUY922 on radiosensitivity between cancer cells and cancer stem cell-like cells.

\section{Materials and methods}

Cell culture. We used the human glioblastoma cell line, T98G (provided by JCRB, Setagaya, Tokyo), in the present 
study. T98G cells were cultured in $\alpha$-minimum essential medium ( $\alpha$-MEM) supplemented with $20 \mathrm{mM}$ 4-(2-hydroxyethyl) piperazine ethane sulfonic acid, $8 \mathrm{mM} \mathrm{NaHCO}_{3}$, $50 \mu \mathrm{g} / \mathrm{ml}$ streptomycin, $50 \mathrm{U} / \mathrm{ml}$ penicillin, and $10 \%$ fetal calf serum and maintained in a humidified incubator at $37^{\circ} \mathrm{C}$ containing a mixture of $98 \%$ air and $2 \% \mathrm{CO}_{2}$.

Spheroid culture. T98G cells were seeded onto non-adherent U-shaped bottom 96-well plates (PrimeSurface 96U, MS-9096U,Sumitomo Bakelite Co.,Ltd.). The seeded cells were cultured in $\alpha$-MEM at a density of 5,000 or 10,000 cells/well for 3 days at $37^{\circ} \mathrm{C}$ under conditions of $98 \%$ air and $2 \% \mathrm{CO}_{2}$. The seeded cells aggregated and formed a cell mass at the bottom of the nonadherent U-shaped wells on the plates. The spheroids formed were transferred to non-adherent 100-mm dishes (PrimeSurface 100Ф, MS-9090X, Sumitomo Bakelite Co., Ltd.) at a density of 96 spheroids/dish after 3 days of culture. The transferred spheroids were then successively cultured for 7-10 days until they reached a diameter of 300-500 $\mu \mathrm{m}$.

Preparation of frozen cryostat sections. After the spheroids were fixed with a solution containing $10 \%$ formalin and $10 \%$ sucrose for $1 \mathrm{~h}$, the spheroids were rinsed with phosphatebuffered saline (PBS) (-). The spheroids were then embedded in Tissue-Tek O.C.T. Compound (Sakura Finetechnical Co., Ltd.) and sliced into frozen sections $10-20 \mu \mathrm{m}$ in thickness using a cryostat.

Immunofluorescence staining. Cryostat sections on glass slides were incubated for double immunofluorescent staining using anti-CD133 ${ }^{\mathrm{AC} 133}$ monoclonal antibody (Miltenyi Biotechnology; 1:10 dilution), anti-HIF-1 $\alpha$ polyclonal antibody (Novus Biologicals; 1:100 dilution), or anti-nestin polyclonal antibody (Santa Cruz Biotechnology; 1:50 dilution) for $1 \mathrm{~h}$ at room temperature. Subsequently, the sections were washed thrice using PBS (-) and then incubated with Alexa Fluor 488 anti-rabbit and Alexa Fluor 546 anti-mouse secondary antibodies (Nacalai Tesque; 1:1,000 dilution) for $1 \mathrm{~h}$ at $37^{\circ} \mathrm{C}$. After washing with PBS (-), the sections were embedded with SlowFade Gold antifade reagent containing 4,6-diamidino-2-phenylindole (Invitrogen; Thermo Fisher Scientific, Inc.), and covered with a glass coverslip. For immunofluorescent staining of monolayer cultured cells, cells were seeded onto a glass slide and cultured for 1 day at $37^{\circ} \mathrm{C}$ with a mixture of $98 \%$ air and $2 \% \mathrm{CO}_{2}$. After 1 day, the cells were washed with PBS (-), fixed with $10 \%$ formalin for $1 \mathrm{~h}$, and then incubated for double immunofluorescent staining using the same method used for cryostat section staining.

Fluorescence-activated cell sorting. After culturing the spheroids for 7-10 days, the spheroids were rinsed once with PBS (-) and then treated with $0.025 \%$ trypsin for $5 \mathrm{~min}$ at $37^{\circ} \mathrm{C}$. Trypsin-treated spheroids were dispersed into single cells via pipetting. The single cells were centrifuged at $1,200 \mathrm{xg}$ for $5 \mathrm{~min}$, rinsed thrice using PBS (-), and treated for 10 min with a PE-labeled anti-CD133 monoclonal antibody (Miltenyi Biotechnology 1:10 dilution) and 7-aminoactinomycin D (7-AAD; 1:10 dilution) for dead cell-labeling. PBS containing $1 \%$ bovine serum albumin (BSA) was used for dilution. Subsequently, the single cells were centrifuged, rinsed thrice with PBS (-), and suspended in PBS containing $1 \%$ BSA. BD FACSJazz cell sorter (BD Biosciences) was used for flow cytometry analysis. Finally, CD133-positive and 7-AAD-negative cells or CD133-negative and 7-AAD-negative cells were sorted as SCPCs and SCNCs, respectively, according to the manufacturer's protocol (BD Biosciences). The clonogenic cell survival assay was performed within $1 \mathrm{~h}$ of sorting the cells.

Clonogenic cell survival assay. The proportion of surviving cells was measured using a colony formation assay. Exponentially growing cells were plated at a density of $1.0 \times 10^{3}$ or $3.0 \times 10^{3}$ cells in three replicate 6 -well plates. These cells were then incubated at $37^{\circ} \mathrm{C}$ for $3 \mathrm{~h}$ in a $\mathrm{CO}_{2}$ incubator. Subsequently, the cells were irradiated in $\alpha$-MEM with $150 \mathrm{kV}$ $\mathrm{X}$-rays emitted by an irradiator (Softex, M-150ME) at a dose rate of $1.1 \mathrm{~Gy} / \mathrm{min}$ under normoxia at room temperature. Non-irradiated cells were treated with 0,10 , and $50 \mathrm{nM}$ of NVP-AUY922 (Novartis) or dimethyl sulfoxide (DMSO) for $24 \mathrm{~h}$, following which the medium containing NVP-AUY922 or DMSO was replaced with a NVP-AUY922-free or DMSO-free medium. We used doses of 10 and $50 \mathrm{nM}$ of NVP-AUY922 on the basis of pre-experiments. Under combined treatment with X-rays and NVP-AUY922, the cells were irradiated in $\alpha$-MEM containing NVP-AUY922 or DMSO. $\alpha$-MEM containing NVP-AUY922 or DMSO was replaced with a NVP-AUY922- or DMSO-free medium $24 \mathrm{~h}$ after irradiation. The cells were subsequently incubated at $37^{\circ} \mathrm{C}$ for $10-14$ days in a $\mathrm{CO}_{2}$ incubator. The colonies formed were stained with crystal violet and dissolved in $20 \%$ methanol. Colonies containing more than 50 cells were counted as survivors. Three independent experiments were performed.

Statistical analysis. To analyze a significant interaction effect, two-way ANOVA followed by a post hoc Tukey's HSD test was conducted. Paired Student's t-test was also used for confirmation of the difference between CD133-positive and CD133-negative cells. $\mathrm{P}<0.01$ was considered to indicate a statistically significant difference.

\section{Results}

Immunofluorescent double-staining of CD133 ${ }^{A C 133}$ and $H I F-1 \alpha$. Fig. $2 \mathrm{~A}-\mathrm{F}$ shows anti-CD133 ${ }^{\mathrm{AC} 133}$ and anti-HIF-1 $\alpha$ antibody immunofluorescence double-stained images. In monolayer cultured cells (Fig. 2A-C), only a few CD133-or HIF-1 $\alpha$-positive cells were observed occasionally. In contrast, in the cryostat sections from spheroids (Fig. 2D-F), CD133 positive or HIF-1 $\alpha$ positive cells were observed. CD133 positive cells were also positive for HIF-1 $\alpha$ in the hypoxic region, as suggested by HIF-1 $\alpha$ positivity (Fig. $2 \mathrm{~F}$ ).

Immunofluorescent double-staining of CD133 $3^{A C 133}$ and nestin. Fig. 2G-L shows CD133 ${ }^{\mathrm{AC} 133}$ and nestin (a marker for undifferentiated neural cell) antibodies double-stained immunofluorescence images. In monolayer cultured cells (Fig. 2G-I), only a few CD133- and nestin-positive cells were observed occasionally. In contrast, CD133 positive or nestin-positive cells were observed in the cryostat sections from spheroids (Fig. 2J-L). CD133 positive cells were also 


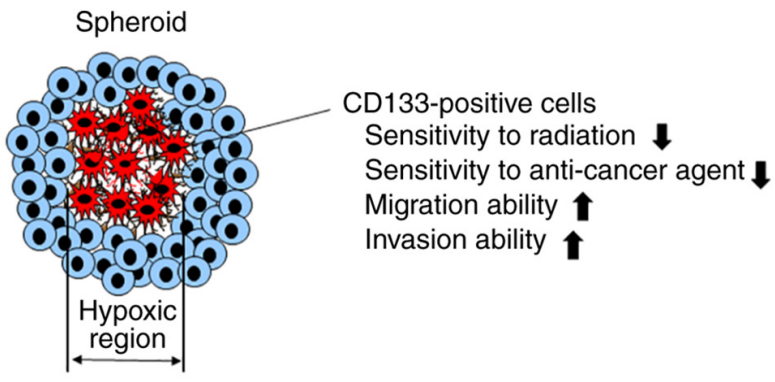

Figure 1. A schema on significance of CD-133 positive cells. The figure shows cancer stem cell-like properties in CD133-positive cells in T98G cell line-derived spheroids.

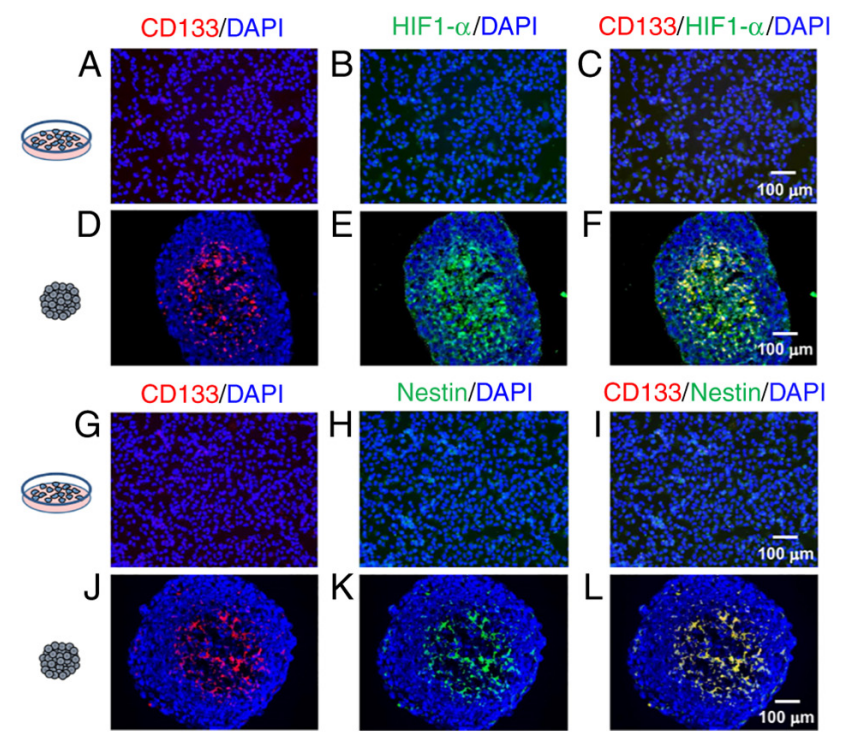

Figure 2. Images of CD133/HIF-1 $\alpha$ immunofluorescent double staining. Monolayer cultured cells staining for (A) CD133, (B) HIF-1 $\alpha$ and (C) CD133/HIF-1 $\alpha$; spheroid sections on day 10 after cell seeding for (D) CD133 (red-colored), (E) HIF-1 $\alpha$ (green-colored) and (F) CD133/HIF-1 $\alpha$ (yellow-colored). Images of CD133/nestin immunofluorescence double staining: Monolayer cultured cells staining for $(\mathrm{G}) \mathrm{CD} 133$, (H) nestin and (I) CD133/nestin; spheroid sections on day 10 after cell seeding for (J) CD133 (red-colored), (K) nestin (green-colored) and (L) CD133/nestin (yellow-colored). HIF, hypoxia-inducible factor- $1 \alpha$.

positive for nestin and were observed in the central region of spheroids (Fig. 2L).

Cell sorting of the CD133-positive and CD133-negative cells of spheroids. Single cells that were dispersed from spheroids and stained immunofluorescently with anti-CD133AC133 antibodies were analyzed. CD133-positive and 7-AAD-negative cells (red-colored gating) represented $\sim 0.8 \%$ of all measured single cells in monolayer cultured cells (negative control; Fig. 3A and C) and $\sim 15 \%$ in the cells from spheroids (Fig. 3B and D). Sorted CD133-positive and 7-AAD-negative cells or CD133-negative and 7-AAD-negative cells (blue-colored gating) from spheroids were used for the cell viability assay.

Viability of SCPCs and SCNCs as determined using colony-formation assay. SCPCs and SCNCs sensitivity to X-rays (6 Gy), NVP-AUY922 (10 and $50 \mathrm{nM}$ ), or X-rays
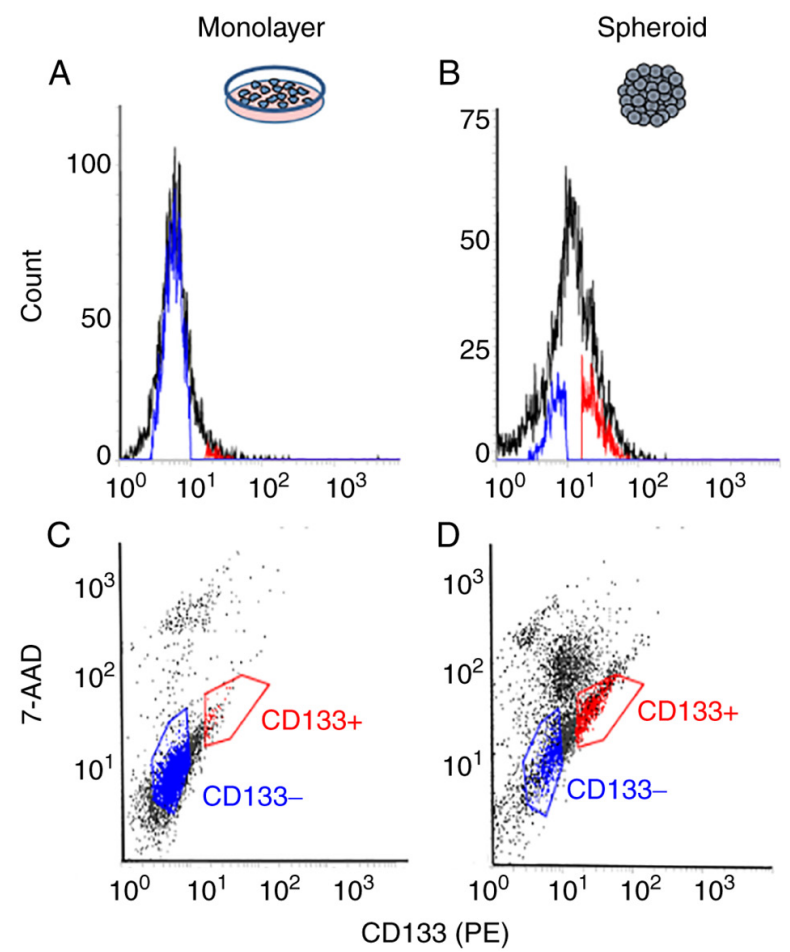

Figure 3. Gating of CD133-positive and CD133-negative cells. Histograms on PE (labeled to CD133 antibody) signals of (A) monolayer-cultured cells and (B) spheroid-cultured cells. Cell distributions based on PE and 7-AAD signals from (C) monolayer-cultured cells and (D) spheroid-cultured cells. Spheroid or monolayer CD133-positive cells (red-colored) and spheroid CD133-negative cells (blue-colored) were sorted from the gating shown in $(\mathrm{C}$ and $\mathrm{D})$, respectively. The gating was set on the basis of microscopic observation of PE signals in living cells (7-AAD-negative cells). 7-AAD, 7-aminoactinomycin D.

combined with NVP-AUY922 was examined using a colony-formation assay. As shown in Fig. 4, the survival fractions among SCPCs groups (X-ray 6 Gy $(0.41 \pm 0.021)$ vs. AUY $10 \mathrm{nM}(0.53 \pm 0.025)$ or $50 \mathrm{nM}(0.57 \pm 0.035)$, X-ray 6 Gy vs. X-ray 6 Gy + AUY $10 \mathrm{nM}(0.26 \pm 0.015)$ or $50 \mathrm{nM}(0.15 \pm 0.040)$, AUY 10 or $50 \mathrm{nM}$ vs. X-ray 6 Gy + AUY 10 or $50 \mathrm{nM}$ ) were significantly different $(\mathrm{P}<0.01$, Tukey's HSD) except for the survival fraction between groups of AUY $10 \mathrm{nM}$ and AUY $50 \mathrm{nM}$ with no significant difference $(\mathrm{P}>0.05)$. In the case of SCNCs, the survival fractions among groups (X-ray 6 Gy $(0.24 \pm 0.006)$ vs. X-ray 6 Gy + AUY $10 \mathrm{nM}(0.12 \pm 0.006)$ or $50 \mathrm{nM}(0.11 \pm 0.040)$, AUY $10 \mathrm{nM}(0.27 \pm 0.025)$ or $50 \mathrm{nM}$ $(0.28 \pm 0.010)$ vs. X-ray $6 \mathrm{~Gy}+\mathrm{AUY} 10$ or $50 \mathrm{nM})$ were significantly different $(\mathrm{P}<0.01)$ but those among groups $(\mathrm{X}$-ray $6 \mathrm{~Gy}$ vs. AUY 10 or $50 \mathrm{nM}$, AUY $10 \mathrm{nM}$ vs. AUY $50 \mathrm{nM}$, X-ray 6 Gy + AUY10 nM vs. X-ray 6 Gy + AUY 50 nM) were not significantly different $(\mathrm{P}>0.05)$. The difference in the proportion of surviving SCPCs between X-ray $6 \mathrm{~Gy}$ and X-ray $6 \mathrm{~Gy}+$ AUY 50 nM (Cohen's d=6.22, effect size) was larger compared with that of surviving SCNCs between X-ray 6 Gy and X-ray $6 \mathrm{~Gy}+$ AUY $50 \mathrm{nM}(\mathrm{d}=4.50)$. In addition, the difference in the proportion of surviving SCPCs between AUY $10 \mathrm{nM}(\mathrm{d}=8.79)$ or $50 \mathrm{nM}(\mathrm{d}=8.54)$ and X-ray $6 \mathrm{~Gy}+\mathrm{AUY} 50 \mathrm{nM}$ was larger compared with that of surviving SCNCs between AUY $10 \mathrm{nM}$ $(\mathrm{d}=4.85)$ or $50 \mathrm{nM}(\mathrm{d}=5.66)$ and X-ray $6 \mathrm{~Gy}+$ AUY $50 \mathrm{nM}$. The larger Cohen's d values in SCPCs indicated that the sensitivity of SCPCs to X-rays or NVP-AUY was more strongly 


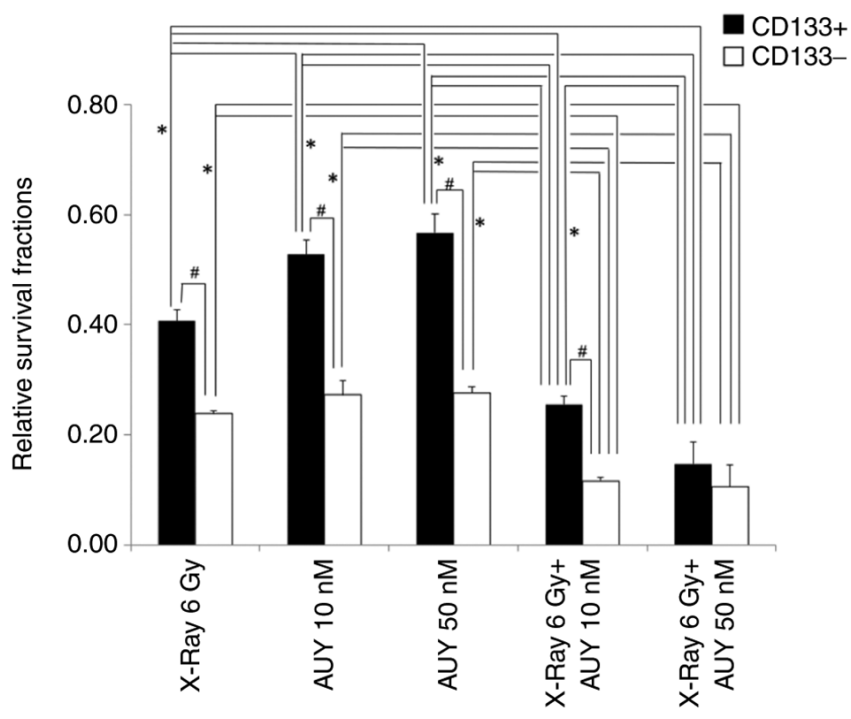

Figure 4. Surviving fractions of SCPCs and SCNCs. SCPCs (CD133+; black columns) and SCNCs (CD133; white columns) sorted from T98G spheroids were irradiated with X-rays, treated with N-vinylpyrrolidone-AUY922 alone or combined with X-rays. All plots represent the means and standard deviations of at least three independent experiments. " $\mathrm{P}<0.01$ (Tukey's HSD test); ${ }^{\text {P }}<0.01$ (Student's t-test). SCPCs, spheroid CD133-positive cells; SCNCs, spheroid CD133-negative cells.

enhanced by combined treatment of X-rays and NVP-AUY than that of SCNCs.

Since the interaction was significant, we also confirmed the difference between CD133-positive and CD133-negative cells. The survival fractions of SCPCs exposed to X-ray 6 Gy, AUY $10 \mathrm{nM}$, and AUY $50 \mathrm{nM}$ were significantly larger $(\mathrm{P}<0.01$, Student's t-test) than those of SCNCs exposed to X-ray $6 \mathrm{~Gy}$, AUY $10 \mathrm{nM}$, and AUY $50 \mathrm{nM}$, respectively, under X-ray irradiation or NVP-AUY922 treatment conditions, indicating a lower sensitivity of SCPCs to X-rays and NVP-AUY922. Under treatment with X-ray 6 Gy irradiation combined with a low concentration of AUY (10 nM), a significant difference in the proportion of surviving SCPCs and SCNCs was observed $(\mathrm{P}<0.01)$ but that of surviving SCPCs and SCNCs was not observed $(\mathrm{P}>0.05)$ under treatment with X-ray $6 \mathrm{~Gy}$ irradiation combined with a high concentration of AUY (50 nM). We did not use other HSP90 inhibitors because it was reported that other inhibitors have high cell toxicity (15-17).

\section{Discussion}

The present study confirmed the findings of our previous study, indicating that glioblastoma SCPCs are induced in the hypoxic microenvironment of T98G cell line-derived spheroids, and they are positive for nestin, which is a marker for undifferentiated neural cells (7). The sensitivity of SCPCs to X-rays is lower than that of SCNCs and migration and inversion abilities of SCPCs are higher than those of SCNCs (9). Therefore, we used T98G cell line-derived SCPCs as the CSC models in the present study. We have not yet analyzed the CSC-like properties of SCPCs in other glioblastoma cell lines. The significance of SCPCs is shown in Fig. 1. In addition, we reported that CD133-positive cells are not induced under hypoxia in monolayer cell culture condition (9). It seems that a combined condition of two dimensional culture and hypoxia is not enough for the induction of stem cell-like cells.

Hsp90 protects against the degradation of numerous client proteins that contribute to the maintenance of cancer cells. Both SCPCs and SCNCs were sensitive to the Hsp90 inhibitor NVP-AUY922, while the sensitivity of SCPCs to NVP-AUY922 was lower than that of SCNCs, as shown in Fig. 4. In general, cancer stem cells are resistant to anti-cancer agents; thus, SCPCs may show lower sensitivity to NVP-AUY922. The resistance of cancer stem cells to NVP-AUY922 has not yet been reported elsewhere.

Inhibition of Hsp90 by NVP-AUY922 has been reported to induce radiosensitization of cancer cells $(13,20-22)$. In the present study, radiosensitization by NVP-AUY922 was observed in glioblastoma SCPCs and SCNCs. Interestingly, the sensitivity of SCPCs to radiation was more strongly enhanced dose-dependently by NVP-AUY922 than that of SCNCs, as shown in Fig. 4. This result suggests that NVP-AUY922 may cause preferential radiosensitization of glioblastoma cancer stem cells. The preferential radiosensitization has not yet been reported in other Hsp90 inhibitors. There are possible mechanisms for the preferential radiosensitization of SCPCs by NVP-AUY922. NVP-AUY922 may inhibit some cancer stem cell properties, such as enhanced DNA repair $(3,5)$, removal of reactive oxygen species (23), and activation of Akt-mediated cell survival signal transduction (24). In addition, NVP-AUY922 may inhibit client proteins that maintain cancer cell stemness. Hypoxia-inducible factor-1 $\alpha$ (HIF-1 $\alpha$ ) is an Hsp90 client protein that plays important roles in maintaining cancer cell stemness. Previous studies using tumor cells in patients and established cancer cell lines showed that HIF-1 $\alpha$ maintains glioma stem cells $(25,26)$, promotes glioma stem cell expansion (27), and upregulates CD133 protein expression $(28,29)$. Furthermore, HIF-1 $\alpha$ may mediate the expression of chemoresistance markers in CSCs (30). From these reports, it is assumed that HIF-1 $\alpha$ inhibition by NVP-AUY922 might induce higher sensitization of cancer stem cells to radiation. In the present study, we did not examine effects of NVP-AUY922 on cancer stem cell markers such as HIF-1 $\alpha$, CD133, nestin and others. It is important to explore the mechanism of preferential radiosensitization by NVP-AUY922 in glioblastoma cancer stem cells through analyses of the location or expression of the markers in spheroids.

In summary, NVP-AUY922 enhanced the sensitivity to radiation more strongly in SCPCs than in SCNCs sorted from T98G cell line-derived spheroids. The present study suggests for the first time that an Hsp90 inhibitor, NVP-AUY922, is a candidate for preferential radiosensitization in glioblastoma cancer stem cells.

\section{Acknowledgements}

The authors would like to thank Professor Kohichi Iwai (Ibaraki Prefectural University of Health Sciences, Ibaraki, Japan) for their helpful comments regarding the statistical analyses.

\section{Funding}

This work was supported by Grants-in-Aid from the Ministry of Education, Science, Sports, Culture and Technology of Japan (grant no. 16K10399) and a subsidy for Science and Technology 
Promotion of Prefectures Locating Electric Power Plants from the Ministry of Education, Culture, Sports, Science and Technology of Japan to Ibaraki Prefectural University of Health Sciences.

\section{Availability of data and materials}

The datasets used and/or analyzed during the current study are available from the corresponding author on reasonable request.

\section{Authors' contributions}

TT performed histological and cytological examination using cell lines and spheroids and was a major contributor in writing the manuscript. NT participated in cell culture and data analysis. $\mathrm{KO}$ contributed to the conceptualization, methodology, review and editing of the manuscript. TT, NT, and KO confirm the authenticity of all the raw data. All authors read and approved the final manuscript.

\section{Ethics approval and consent to participate}

Not applicable.

\section{Patient consent for publication}

Not applicable.

\section{Competing interests}

The authors declare that they have no competing interests.

\section{References}

1. Stupp R, Hegi ME, Mason WP, van den Bent MJ, Taphoorn MJ, Janzer RC, Ludwin SK, Allgeier A, Fisher B, Belanger K, et al: Effects of radiotherapy with concomitant and adjuvant temozolomide versus radiotherapy alone on survival in glioblastoma in a randomised phase III study: 5-year analysis of the EORTC-NCIC trial. Lancet Oncol 10: 459-466, 2009.

2. Singh SK, Hawkins C, Clarke ID, Squire JA, Bayani J, Hide T, Henkelman RM, Cusimano MD and Dirks PB: Identification of human brain tumour initiating cells. Nature 432: 396-401, 2004.

3. Bao S, Wu Q, McLendon RE, Hao Y, Shi Q, Hjelmeland AB, Dewhirst MW, Binger DD and Rich JN: Glioma stem cells promote radioresistance by preferential activation of the DNA damage response. Nature 444: 756-760, 2006.

4. Beier D, Schulz JB and Beier CP: Chemoresistance of glioblastoma cancer stem cells-much more complex than expected. Mol Cancer 10: 128, 2011.

5. Mannino M and Chalmers AJ: Radioresistance of glioma stem cells: Intrinsic characteristic or property of the "microenvironment-stem cell unit'? Mol Oncol 5: 374-386, 2011.

6. Lathia JD, Mack SC, Mulkearns-Hubert EE, Valentim CL and Rich JN: Cancer stem cells in glioblastoma. Genes Dev 29: 1203-1217, 2015.

7. Ohnishi K, Tani T, Bando S, Kubota N, Fujii Y, Hatano O and Harada H: Plastic induction of CD133AC133-positive cells in the microenvironment of glioblastoma spheroid. Int J Oncol 45 : 581-586, 2014.

8. Sutherland RM: Cell and environment interactions in tumor microregions: The multicell spheroid model. Science 240: 177-184, 1988.

9. Ohnishi K, Tani T, Tojo N and Sagara JI: Glioblastoma cell line shows phenotypes of cancer stem cells in hypoxic microenvironment of spheroids. Biochem Biophys Res Commun 546: 150-154, 2021.

10. Trepel J, Mollapour M, Giaccone G and Neckers L: Targeting the dynamic HSP90 complex in cancer. Nat Rev Cancer 10: 537-549, 2010.
11. Yuno A, Lee MJ, Lee S, Tomita Y, Rekhtman D, Moore B and Trepel JB: Clinical evaluation and biomarker profiling of Hsp90 inhibitors. Methods Mol Biol 1709: 423-441, 2018.

12. Dote H, Burgan WE, Camphausen K and Tofilon PJ: Inhibition of hsp90 compromises the DNA damage response to radiation. Cancer Res 66: 9211-9220, 2006

13. Camphausen K and Tofilon PJ: Inhibition of Hsp90: A multitarget approach to radiosensitization. Clin Cancer Res 13: 4326-4330, 2007.

14. Jhaveri K, Taldone T, Modi S and Chiosis G: Advances in the clinical development of heat shock protein 90 (Hsp90) inhibitors in cancers. Biochim Biophys Acta 1823: 742-755, 2012.

15. Gaspar N, Sharp SY, Pacey S, Jones C, Walton M, Vassal G, Eccles S, Pearson A and Workman P: Acquired resistance to 17-allylamino-17-demethoxygeldanamycin (17-AAG, tanespimycin) in glioblastoma cells. Cancer Res 69: 1966-1975, 2009.

16. Piper PW and Millson SH: Mechanisms of resistance to Hsp90 inhibitor drugs: A complex mosaic emerges. Pharmaceuticals (Basel) 4: 1400-1422, 2011.

17. Jarosz D: HSP90: A global regulator of the genotype-to-phenotype map in cancers. Adv Cancer Res 129: 225-247, 2016.

18. Lee KH, Lee JH, Han SW, Im SA, Kim TY, Oh DY and Bang YJ: Antitumor activity of NVP-AUY922, a novel heat shock protein 90 inhibitor, in human gastric cancer cells is mediated through proteasomal degradation of client proteins. Cancer Sci 102: 1388-1395, 2011.

19. Gaspar N, Sharp SY, Eccles SA, Gowan S, Popov S, Jones C, Pearson A, Vassal G and Workman P: Mechanistic evalution of the novel HSP90 inhibitor NVP-AUY92 in adult and pediatric glioblastoma. Mol Cancer Ther 9: 1219-1233, 2010.

20. Zaidi S, McLaughlin M, Bhide SA, Eccles SA, Workman P, Nutting CM, Huddart RA and Harrington KJ: The HSP90 inhibitor NVP-AUY922 radiosensitizes by abrogation of homologous recombination resulting in mitotic entry with unresolved DNA damage. PLoS One 7: e35436, 2012.

21. Gandhi N, Wild AT, Chettiar ST, Aziz K, Kato Y, Gajula RP, Williams RD, Cades JA, Annadanam A, Song D, et al: Novel Hsp90 inhibitor NVP-AUY922 radiosensitizes prostate cancer cells. Cancer Biol Ther 14: 347-356, 2013.

22. Hashida S, Yamamoto H, Shien K, Ohtsuka T, Suzawa K, Maki Y, Furukawa M, Soh J, Asano H, Tsukuda K, et al: Hsp90 inhibitor NVP-AUY922 enhances the radiation sensitivity of lung cancer cell lines with acquired resistance to EGFR-tyrosine kinase inhibitors. Oncol Rep 33: 1499-1504, 2015.

23. Diehn M, Cho RW, Lobo NA, Kalisky T, Dorie MJ, Kulp AN, Qian D, Lam JS, Ailles LE, Wong M, et al: Association of reactive oxygen species levels and radioresistance in cancer stem cells. Nature 458: 780-783, 2009

24. Man J, Shoemake JD, Ma T, Rizzo AE, Godley AR, Wu Q, Mohammadi AM, Bao S, Rich JN and Yu JS: Hyperthermia sensitizes glioma stem-like cells to radiation by inhibiting AKT signaling. Cancer Res 75: 1760-1769, 2015.

25. Heddleston JM, Li Z, McLendon RE, Hjelmeland AB and Rich JN: The hypoxic microenvironment maintains glioblastoma stem cells and promotes reprogramming towards a cancer stem cell phenotype. Cell Cycle 8: 3274-3284, 2009.

26. Mao XG, Yan M, Xue XY, Zhang X, Ren HG, Guo G, Wang P, Zhang W and Huo JL: Overexpression of ZNF217 in glioblastoma contributes to the maintenance of glioma stem cells regulated by hypoxia-inducible factors. Lab Invest 91: 1068-1078, 2011.

27. Soeda A, Park M, Lee D, Mintz A, Androutsellis-Theotokis A, McKay RD, Engh J, Iwama T, Kunisada T, Kassam AB, et al: Hypoxia promotes expansion of the CD133-positive glioma stem cells through activation of HIF-1alpha. Oncogene 28: 3949-3959, 2009.

28. Bar EE, Lin A, Mahairaki V, Matsui W and Eberhart CG: Hypoxia increases the expression of stem-cell markers and promotes clonogenicity in glioblastoma neurospheres. Am J Pathol 177: 1491-1502, 2010.

29. Iida H, Suzuki M, Goitsuka R and Ueno H: Hypoxia induces CD133 expression in human lung cancer cells by up-regulation of OCT3/4 and SOX2. Int J Oncol 40: 71-79, 2012.

30. Kolenda J, Jensen SS, Aaberg-Jessen C, Christensen K, Andersen C, Brünner N and Kristensen BW: Effects of hypoxia on expression of a panel of stem cell and chemoresistance markers in glioblastoma-derived spheroids. J Neurooncol 103: 43-58, 2011. 